\title{
Whole Blood Screening of Antibodies using Label-free Nanoparticle Biophotonic Array Platform
}

Rouslan V. Olkhov, Robert Parker, ${ }^{1}$ Andrew M. Shaw ${ }^{*}$

College of Life and Environmental Sciences, University of Exeter, Exeter, EX4 4QD, United Kingdom

\begin{abstract}
A gold nanoparticle, localised plasmon array biosensor using light scattering has been employed in the detection of allergen-specific antibodies in whole blood and sera. The array sensor was functionalized with four different allergens, cat dander (Fel d1), dust mite (Der p1), peanut allergen (Ara h1) and dog dander (Can f1) and immuno-kinetic assay was performed to detect their respective anti-allergen IgG antibodies. Specific positive responses to antibodies at a concentration of $25 \mathrm{nM}$ were observed for Fel d1, Der p1, and Ara $\mathrm{h} 1$ allergens, while the Can $\mathrm{f} 1$ channel served as a reference control. The sensitivity was further enhanced using a secondary anti-IgG detection antibodies to give a limit of detection of $2 \mathrm{nM}$. The results indicate the potential for nanoparticle scattering multiplexed arrays to screen unprepared blood samples at point-of-care for assays of complex samples such as the whole blood.
\end{abstract}

\section{Keywords:}

serum, blood, imaging array biosensor, light scattering, immuno-kinetic assay

${ }^{1}$ Present address: Key Organics Ltd., Highfield Industrial Estate, Camelford, PL32 9RA, UK

* Corresponding author: tel: 01392 723495, fax: 01392 723434, email: andrew.m.shaw@exeter.ac.uk 


\section{Introduction}

Microarray biosensors perform very well when purified biomolecular analytes are studied. However, in order for biosensors to become a reliable point-of-care technology in medical diagnostics, they must maintain workable sensitivity and specificities towards target analytes, in media containing a rich complexity of background proteins and cells (Anderson and Anderson 2002). Similar concerns are associated with complex sample analysis in proteomics studies (Panchaud et al. 2008). Several techniques have been developed to address the non-specific protein background, although they typically rely on labelled detection schemes (Borrebaeck 2000; Haab et al. 2001; Hirsch et al. 2003). Similarly, the alternative label-free technologies such as surface plasmon resonance (SPR) or localised surface plasmon resonance (LSPR) surfer from sensor surface instabilities associated with non-specific binding (NSB) from blood derived samples (Luong et al.). Some recent advances in complex fluids sampling have been successful (Billakanti et al. 2010; Gonzales et al. 2002; Mytych et al. 2009), including multiplexed imaging SPR of sera samples (Lausted et al. 2008). In some cases the sample pre-treatment, protein depletion or chaotrope addition, may reduce non-specific contribution to the background (Hifumi et al. 2002; Richens et al. 2009), this approach is highly problematic as it may affect the specific responses as well. The healthcare objective however would be a point-of-care device which requires no sample preparation and has the potential to assay a number of different blood biomarkers simultaneously.

An on-going focus in the healthcare sector is the diagnosis and treatment of allergy and food intolerance (Isolauri et al. 2004). Blood tests for allergy conventionally measure the concentration of the specific lgE in the serum derived after coagulation of the blood but it is also reported that other immunoglobulins, like $\lg G$ and $\lg A$ may play their role in allergic response of a patient (Pleass et al. 2007; Shreffler et al. 2005). Also, serological and skin-prick test results could be rather inconsistent (Niederberger et al. 2001) and false negative or positive results are common. A whole blood immune profile may be more characteristic and at least complement the specific $\lg E$ tests. 
We reported the application of the array format light scattering biosensor for immune kinetic detection of biomolecules in relatively simple samples, either purified or diluted in buffers containing near physiological concentrations of human serum albumin (Jansen van Vuuren et al. 2010; Olkhov and Shaw 2010); in this paper we extend the analytes to include whole blood samples. The array format of the biosensor allows a variety of control data to be recorded in parallel with the target response. The types of controls may include non-specific channels serving to compensate for the effect of bulk sample properties, such as changes in temperature or refractive index, or more specialized to discriminate between false positive or false negative responses resulting from NSB interactions.

\section{Meterial and Methods}

\subsection{Chemicals}

The following chemicals were used: bovine serum albumin, BSA; human fibrinogen, FBR; purified allergens Fel d1 (cat), Can f1 (dog), Der p1 (dust mite), and Ara h1 (peanut); antibodies to Der p1, anti-Der-p1-IgG; antibodies to Fel d1, anti-Fel-d1-IgG; rabbit antibodies to Ara h1, anti-Ara-h1-lgG; polyclonal antibodies to BSA, anti-BSA; antibodies to rabbit IgG, anti-lgG; dithiobis-succinimidyl propionate, DTSP. The phosphate buffed saline buffer containing Tween 20 surfactant, PBS, was used in preparation and dilution of the samples. The aqueous $100 \mathrm{mM}$ phosphoric acid solution was used as regeneration buffer. More details are available in the supplementary information.

\subsection{Experimental Methods}

The detailed overview of the localised plasmon light-scattering array readier technology and principles of the general array preparation were reported earlier (Olkhov et al. 2009; Olkhov and Shaw 2010). The dark-field illuminated sensor array is imaged with CCD video camera while the sensor surface is exposed to various analyte solutions (instrument schematic is presented in Figure S1, supplementary information). The sensor array is covered with a flow cell, which directs the studied sample solution over the sensor surface. The sensing areas are formed by gold nanoparticles deposited on the substrate surface, their light-scattering properties depend on the localised particle plasmon and local microscopic environment close to the particle surface. Therefore, if molecules from the sample are deposited on the particle surface, the scattered light intensity changes and this is recorded as evidence of the binding event. The instrument was modified from previous work to add the ability to image the sensor array either through the flow cell, and hence 
through sample, or through the underside of the sensor substrate, eliminating the sample effects: the latter modification proved to be critical for assaying whole, unprocessed blood samples.

A total of 96 gold nanoparticle coated spots of the sensor array were activated with DTSP and functionalized by inkjet printing of five different proteins (Figure S2 and array preparation details in supplementary information) to introduce bio-specificity to the sensor response, fibrinogen served as a non-specific reference, Fel d1 and Can f1 allergens fulfill the role of specificity controls and Der $\mathrm{p} 1$ and Ara h1 allergens produced target specific sensors. The responses from the similar spots were averaged together into signal channels corresponding to each of five proteins. A similar approach was used to produce a FBR and BSA functionalized sensor (not shown) used in assaying sera samples.

Standard techniques employed in clinical laboratories allow simplification of the blood sample composition, nevertheless all blood and sera samples contain large number of different proteins which provide a background against which the specific biomarker should be detected (Anderson and Anderson 2002; Sadana and Chen 1996).

In the course of our experiments, delipidated serum, whole blood, and heparin stabilized blood media were used to prepare the samples. To allow full control over the target biomarker, a non-human $\lg G$ antibody specific to one of the sensor protein channels were spiked into the human-blood derived medium and these samples were tested for the presence of the spiked species. In several measurements the primary sensor response was further enhanced with detection antibodies specific to the spiked target species, an approach similar to a sandwich assay (Huang et al. 2008). The augmentation of the assay with the several reference channels and exploitation of detection antibodies allowed screening of antibodies in whole blood samples.

\section{Results and Discussion}

\subsection{Sera media}

Human sera-based samples spiked with sheep polyclonal aBSA antibodies were assessed as an intermediate step between purified biomarker in buffer solutions and bloodderived samples. 
Binding kinetic measurements for aBSA from spiked buffer were used as a positive control of the printing and sample preparation processes, Figure 1. The specific binding of aBSA to the BSA sensor channels is readily observed in all measurements but both the antibody-antigen association rates and the endpoint sensor maximum load are affected significantly by serum protein load. The total serum protein content leads to: (i) an overall higher bulk refractive index of the sample compared with running PBS buffer; and (ii) nonspecific binding to the sensor surface, both of which contribute to very fast initial kinetic change in sensor responses, Figure $1 \mathrm{~A}$. While (i) is expected to be the same for the test and control channels, the effects of (ii) may be different depending on the nature of the particular protein used as a control reference. In the present case, the FBR channel appears to serve as a good reference for the fast offset from changes in bulk refractive index in the binding kinetics curve, which can then be subtracted from the BSA channel to reveal the specific aBSA-BSA association kinetics contribution to the observed signal change. Panel $B$ of the Figure 1 shows a comparison of FBR-corrected specific binding response curves recorded in assays of whole and delipidated sera samples spiked with $120 \mathrm{nM}$ aBSA and pure PBS buffer containing $50 \mathrm{nM}$ aBSA. It is evident that the observed deposition rates from both sera-based samples are significantly slower than the response recorded from aBSA solution in pure PBS buffer. This reduced rate may be attributed to the high background non-specific binding from the serum protein load. Also, as expected, the delipidated serum has smaller effect on the kinetics compared with whole serum, Figure 1B. The sharp initial spikes visible in both sera sample responses are FBR referencing artifacts, caused by slight fluidic variations across the sensor slide during running buffer to sample exchange in the flow cell.

In order to perform quantitative assays with serum samples, a series of calibration experiments can be performed to take into account the effects of the non-specific protein and fat content. The sensor response and therefore the standard curves are typically nonlinear, although monotonic, Figure S3. A related procedure using the bulk refractive index to correct for non-specific binding was performed in our earlier study of albumin interference on the immuno-kinetic assay (Jansen van Vuuren et al. 2010) either may be used for a quantitative assay. 


\subsection{Blood media}

The main difference between serum samples and whole blood is the presence of the cells which themselves are absorbing and scattering objects and will interfere with any optical measurements. Further, haemoglobin may interfere directly with a typical interrogation wavelength of the nanoparticle plasmons. In order to establish minimise these interference effects, sensor measurements were performed with imaging optics positioned on either side of the sensor - flow cell assembly, Figure 2.

Figure $2 \mathrm{~A}$ shows the un-corrected data collected by imaging the scattered light through the sample bulk layer. The significant noise levels on the kinetic traces in the first 20 minutes may be attributed directly to the presence of the cells in the blood. After the running buffer was re-introduced into the flow cell, the noise returned to its usual level. Panel $B$ shows the analogous un-corrected data imaged through the body of the prism coupling the illumination light to the sensor, and therefore free from majority of the optical interference of blood cells with signal acquisition. In both of these experiments, after approximately 16 minutes the blood cells start to sediment rapidly on the sensor surface visible as a large peak on the sensograms.

Comparison of Figure 1 and Figure 2 demonstrates that FBR channel in the case of blood-derived samples cannot compensate for the non-specific binding as well as in the case of sera based samples: although the FBR channel, Figure 2, is still a good reference to correct baseline for temperature and/or illumination fluctuations. There is a clear discrepancy in the offset upon sample injection which is not corrected following the switch to running buffer. The fouling of the sensor surface with non-specific blood sample components results in considerable quantities of irreversibly bound material in all channels which is not removed during regeneration step. The sensors are reusable but for only 3-4 assay cycles, with a 10-15\% drop in sensitivity per cycle. However, we have demonstrated successfully the survival and sensitivity of the particle plasmon sensing surface in whole blood. We extended the demonstration to include the analysis of four allergen channels with spiked whole-blood samples. Any or all of the channels may be used as a positive control of the target channel for removal of bulk refractive index, temperature and illumination effects. For the chosen IgG concentrations, the primary binding event to the allergen channel from the whole blood was below the detection threshold of the instrument either because it was below the signal-to-noise ratio, Figure $2 \mathrm{~A}$, or uncertain against the 
background subtraction, Figure 2B. A secondary detection step using anti-lgG antibodies was successfully employed to confirm the specific binding in the corresponding sensor channels.

Given that the back imaging was less affected by blood cells preset in the samples, a series of assays were performed in this configuration to confirm the activity and selectivity of the four-allergen sensor arrays. The FBR channel was used as a non-specific reference channel to correct the raw data for bulk properties (temperature, illumination intensity, sample refractive index) and the Can $\mathrm{f} 1$ channel served as a specific reference to control false positive or negative measurements in other allergen channels. The remaining channels Fel d1, Der p1, and Ara h1 have shown specific response when sample spiked with respective anti-allergen antibodies were tested. An example of the sensograms obtained in sequential testing of two samples made of $10 \%$ blood diluted in PBS media, spiked with $25 \mathrm{nM}$ anti-Fel-d1-lgG and anti-Ara-h1-lgG, is given in Figure 3. Panel A presents four allergen channels after FBR reference was subtracted, and panel $B$ shows the same data after Can f1 and reference was subtracted from Fel d1, Ara h1, and Der p1 channels. Both sample injections time zero, 25nM anti-Fel-d1-lgG in $10 \%$ blood in PBS, and 26 minutes, 25nM anti-Ara-h1-lgG in 10\% blood in PBS, lead to the specific binding response in the corresponding allergen channel, additionally the specific response was confirmed with detection of anti-lgG antibody injections at 17 and 50 minutes. As observed in Figure 2 the FBR reference channel does not compensate for the rapid changes in local refractive index perfectly resulting in the observed injection steps at time points, 0 and 26 min. The specific binding contribution to the observed signal changes is typically $1-3 \%$ increasing to $12-34 \%$ following the wash and addition of the detection antibodies. The positive control Cat f1 channel however makes an accurate subtraction, Figure 2B, recovering 100\% sensitivity and specificity for both primary binding and secondary detection steps. Although the spiked anti-allergen-IgG concentrations in both samples were the same, the sensor response to anti-Ara-h1-IgG was much stronger compared with anti-Fel-d1-lgG, this can tentatively be attributed to the fact that anti-Ara-h1-lgG was polyclonal while anti-Fel-d1 - a monoclonal antibody; the latter can only bind to allergens with appropriate configuration on the sensor surface which is not controlled in current experiments. Consequently the detection limits during the primary injection phase are $\mathrm{Ig}$ anaylte dependent: for anti-Ara-h1-IgG the limit of detection is typically $7 \mathrm{nM}$, wheras for 
anti-Fel-d1-lgG the limit of detection is $>25 \mathrm{nM}$. The secondary detection antibodies improve the detection limit to $2 \mathrm{nM}$. The Der $\mathrm{p} 1$ channel in the experiment is a spectator and does not specifically bind either spiked anti-Fel-d1-lgG/anti-Ara-h1-lgG antibodies or detect anti-lgG.

\section{Conclusions}

We have demonstrated that the near-field illumination of gold nanoparticles viewed through the total-internal reflection element allows analysis of the specific response of allergen antibodies in whole blood samples. The specific response was observed towards detection of several anti-allergen IgG spiked in $10 \%$ blood. The multiplexed array format of the sensor allows testing for a number of targets on the same sensor and also permits deployment of several specific and non-specific controls which ensure unambiguous detection of a particular target analyte. The subsequent secondary detection has been demonstrated previously to be quantitative and thus the gold nanoparticle, localised surface plasmon array format has the potential to be a sample preparation-free, point-ofcare technology for the multi-analyte, biomarker profiling of whole blood.

\section{Acknowledgements}

The work was supported by the BBSRC grant BB/FOF/326.

\section{Appendix A. Supplementary data}

Supplementary data is available in the online version, at doi: 

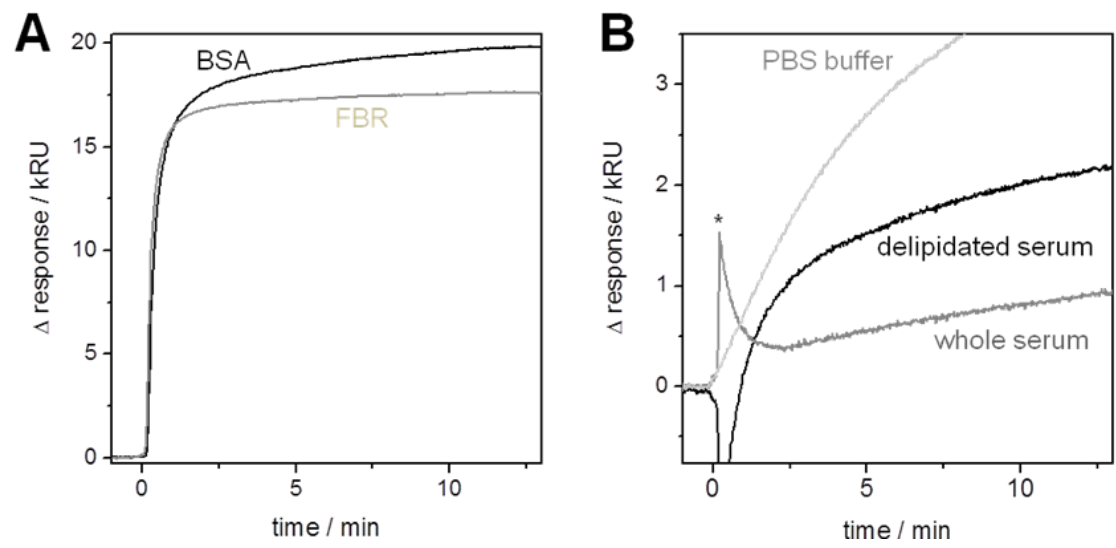

Figure 1. Binding kinetics observed in testing 120nM anti-BSA antibody solutions in buffer and sera media: (A) response of specific BSA functionalised sensor channel (black) against non-specific FBR reference (gray) upon exposure to sample based on delipidated serum, fast initial rise is partly due to bulk refractive index change of the analyte; (B) normalized responses to whole (gray) and delipidated (black) sera samples, light gray curve represents adsorption of $50 \mathrm{nM}$ aBSA in pure PBS buffer for comparison. Responses are referenced with FBR functionalized sensor spots, which resulted in artificial spikes $\left(^{*}\right)$ at the beginning of the traces.
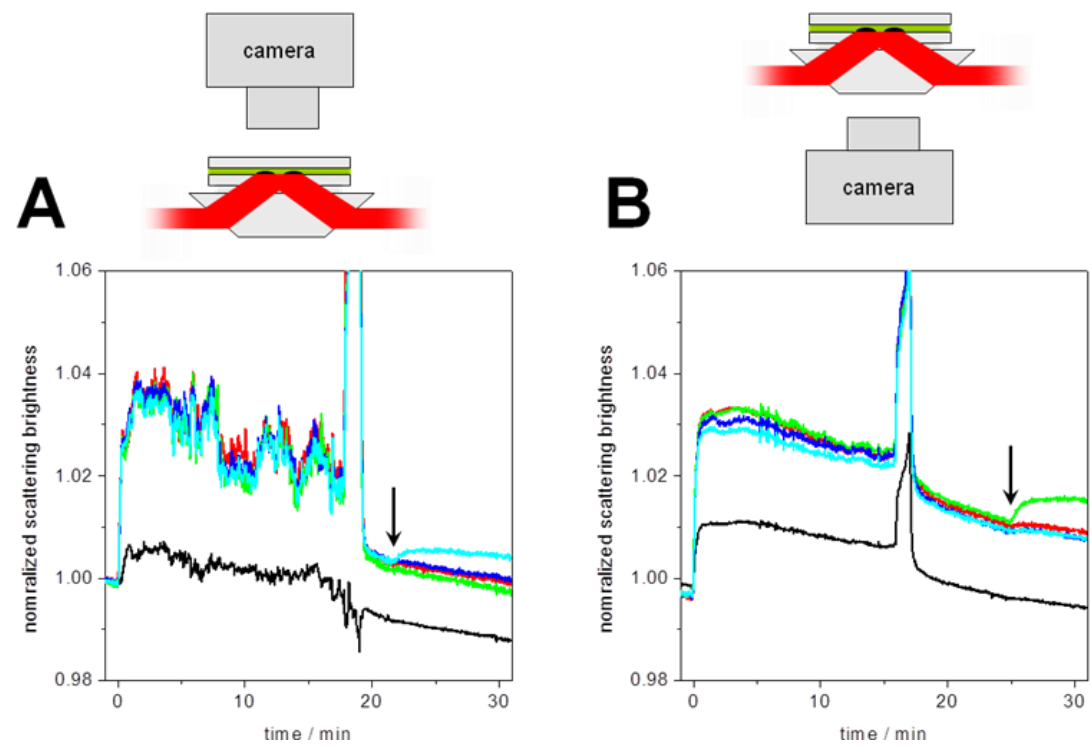

Figure 2. Testing the blood based (10\% blood in PBS) samples with different imaging arrangements: (A) through the bulk of sample, analyte was spiked with anti-Der-P1-IgG, detection anti-IgG was injected at ca. 22 minutes; (B) from the sensor back direction, analyte was spiked with anti-Ara-h1-IgG, detection anti-IgG was injected at ca. 25 minutes. In both cases the specific IgG load is revealed by detection antibody, injection point marked with an arrow. 

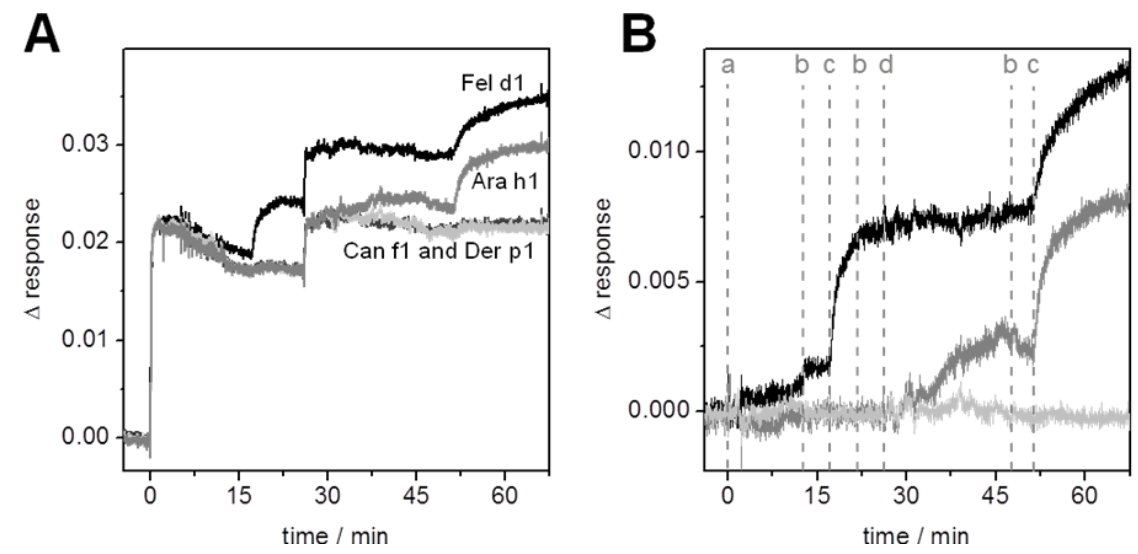

Figure 3. Kinetic assay data measured on four allergen sensor: (A) allergen channels after subtraction of FBR reference, black - Fel d1, gray - Ara h1, light gray - Der p1, dark gray - Can f1; (B) Fel d1 (black), Ara h1 (gray), and Der p1 (light gray) channels additionally referenced against of Can $\mathrm{f1}$ channel. The marked sequence of analyte injections was (a) - anti-Fel-d1-IgG in $10 \%$ blood, (b) - b running buffer, (c) detection anti-lgG, (d) - antiAra-h1-IgG in $10 \%$ blood 


\section{References}

Anderson, N.L., Anderson, N.G., 2002. Mol Cell Proteomics 1(11), 845-867.

Billakanti, J.M., Fee, C.J., Lane, F.R., Kash, A.S., Fredericks, R., 2010. Int. Dairy J. 20(2), 96-105.

Borrebaeck, C.A.K., 2000. Immunol. Today 21(8), 379-382.

Gonzales, N.R., Schuck, P., Schlom, J., Kashmiri, S.V.S., 2002. J. Immunol. Methods 268(2), 197-210.

Haab, B., Dunham, M., Brown, P., 2001. Genome Biol. 2(2), research0004.0001-0004.0013.

Hifumi, E., Kubota, N., Niimi, Y., Shimizu, K., Egashira, N., Uda, T., 2002. Anal. Sci. 18(8), 863-867.

Hirsch, L.R., Jackson, J.B., Lee, A., Halas, N.J., West, J.L., 2003. Anal. Chem. 75(10), 2377-2381.

Huang, H., Ran, P., Liu, Z., 2008. Sensors Actuators B: Chem. 131(2), 417-423.

Isolauri, E., Huurre, A., Salminen, S., Impivaara, O., 2004. Clin. Exp. Allergy 34(7), 1007-1010.

Jansen van Vuuren, B., Read, T., Olkhov, R.V., Shaw, A.M., 2010. Anal. Biochem. 405(1), 114-120.

Lausted, C., Hu, Z., Hood, L., 2008. Mol. Cell. Proteomics 7(12), 2464-2474.

Luong, J.H.T., Male, K.B., Glennon, J.D., Biotechnol. Adv. 26(5), 492-500.

Mytych, D.T., La, S., Barger, T., Ferbas, J., Swanson, S.J., 2009. J. Pharm. Biomed. Anal. 49(2), 415-426.

Niederberger, V., Stubner, P., Spitzauer, S., Kraft, D., Valenta, R., Ehrenberger, K., Horak, F., 2001. J. Invest. Dermatol. 117(4), 848-851.

Olkhov, R.V., Fowke, J.D., Shaw, A.M., 2009. Anal. Biochem. 385, 234-241.

Olkhov, R.V., Shaw, A.M., 2010. Anal. Biochem. 396(1), 30-35.

Panchaud, A., Affolter, M., Moreillon, P., Kussmann, M., 2008. J. Proteomics 71(1), 19.

Pleass, R.J., Lang, M.L., Kerr, M.A., Woof, J.M., 2007. Mol. Immunol. 44(6), 1401-1408.

Richens, J.L., Lunt, E.A.M., Sanger, D., McKenzie, G., O'Shea, P., 2009. J. Proteome Res. 8(11), 5103.

Sadana, A., Chen, Z., 1996. Biosens. Bioelectron. 11(1-2), 17-33.

Shreffler, W.G., Lencer, D.A., Bardina, L., Sampson, H.A., 2005. J. Allergy Clin. Immunol. 116(4), 893-899. 